\title{
Gut microbiome and inflammatory bowel disease (IBD) - is there a link?
}

\author{
Tatiana Grigoryeva \\ KFU, Kazan, Russia \\ tata.grigoryeva@gmail.com \\ Nikolai Ivanov \\ PMI R\&D, Neuchatel, Switzerland \\ nikolai.ivanov@pmi.com
}

Rustem Abdulkhakov KSMU, Kazan, Russia rustemabdul@mail.ru

Mariia Siniagina

KFU, Kazan, Russia

marias25@mail.ru

\author{
Giuseppe Lo Sasso \\ PMI R\&D, Neuchatel, Switzerland \\ Giuseppe.Losasso@pmi.com \\ Manuel Peitsch \\ PMI R\&D, Neuchatel, Switzerland \\ manuel.peitsch@pmi.com \\ Alfiya Odintsova \\ RCH, Kazan, Russia \\ odincovaa@yandex.com \\ Dilyara Khusnutdinova \\ KFU, Kazan, Russia \\ dilyahusn@gmail.com
}

\author{
Julia Hoeng \\ PMI R\&D, Neuchatel, Switzerland \\ Julia.Hoeng@pmi.com \\ Natalya Danilova \\ KFU, Kazan, Russia \\ danilova.natalya.87@mail.ru
}

Maria Markelova

KFU, Kazan, Russia

mimarkelova@gmail.com

Ilya Vasiliev

KFU, Kazan, Russia

mepk_m6@mail.ru

\author{
Sayar Abdulkhakov \\ KFU, Kazan, Russia \\ sayarabdul@yandex.ru
}

\begin{abstract}
The pathogenesis of IBD is not fully known, but one of the factors predisposing to the development of inflammation is probably an imbalance between commensal bacteria and pathogens in the intestinal lumen, a decrease in microbial diversity, and impaired functional metabolism of bacteria. Fecal microbiota whole-genome sequencing confirmed the presence of specific IBD dysbiotic features at the phylum level, with increased abundance of Proteobacteria, Actinobacteria, and Fusobacteria and decrease in Firmicutes, Bacteroidetes, and Verrucomicrobia. At the genus level, the abundance of both fermentative (SCFA-producing and H2releasing) and hydrogenotrophic (H2-consuming) microbes was affected in IBD patients. This imbalance was confirmed by the decreased abundance of SCFA species in the feces of IBD subjects as well as the change in anaerobic index, which mirrors the redox status of the intestine. Conclusion: Our analyses highlighted how IBD-related dysbiotic microbiota- which are generally mainly linked to SCFA imbalance_-might affect other important metabolic pathways, such as $\mathrm{H} 2$ metabolism, that are critical for host physiology and disease development.
\end{abstract}

Keywords - intestinal microbiota, shotgun sequencing, 16SrRNA metagenome, inflammatory bowel diseases

\section{Motivation and aim}

Inflammatory bowel diseases (IBD) are a group of disorders characterized by chronic inflammation of the gastrointestinal tract. IBD present with two main manifestations: ulcerative colitis and Crohn's disease, each having distinctive clinical and pathological features. The etiology of IBD is not fully understood, and the term is used to describe a collection of chronic immune-mediated diseases of unknown, multifactorial etiology with complex interactions between genetic and environmental factors.

Aim of the study revealed differences in microbiome composition among the ulcerative colitis, Crohn's disease, and healthy control subjects.

\section{Methods}

A total of 126 subjects (42 healthy volunteers and 41 Crohn's disease and 43 ulcerative colitis patients) were studied. IBD was diagnosed on the basis of standard clinical, endoscopic, and histological criteria. Fecal microbiota composition was analyzed by $16 \mathrm{~S}$ and whole-genome shotgun sequencing.

\section{Results}

Changes in the intestinal microbiota in patients with UC and $\mathrm{CD}$ are characterized by an increase in the presence of bacteria of the fila Proteobacteria and Bacteroidetes against the background of a decrease in the relative number of bacteria fila Firmicutes and Archaea Euryarchaeota; a decrease in the index of alpha-diversity of bacteria, a decrease in the relative representation of butyrate-producing, hydrogen-utilizing bacteria, Methanobrevibacter smithii, which may be a prerequisite for a violation of colonization resistance. An increase in the relative representation of Ruminococcus gnavus and Akkermansia muciniphila may be an additional, characteristic of patients with $\mathrm{UC}$ and $\mathrm{CD}$, a sign of dysbiosis. A decrease in the representation of the Butyryl-CoA: acetate CoA transferase gene in patients with $\mathrm{CD}$, a decrease in the absolute content of both individual fatty acids and their total amount, and a change in the ratio of the main fatty acids in patients with IBD may indicate a decrease in the functional activity and amount of anaerobic microflora and / or change in utilization of fatty acids by colonocytes. Metabolic dysbiosis associated with an increase in the relative representation of pathways in patients with IBD. The revealed changes can be considered as characteristic shifts in gut microbiome under IBD and potential targets in the selection of personalized therapy. 